\title{
Twenty-Fourth Power Residue Difference Sets
}

\author{
By Ronald J. Evans*
}

\begin{abstract}
It is proved that if $p$ is a prime $\equiv 1(\bmod 24)$ such that either 2 is a cubic residue or 3 is a quartic residue $(\bmod p)$, then the twenty-fourth powers $(\bmod p)$ do not form a difference set or a modified difference set.
\end{abstract}

1. Introduction. Let $p=e f+1$ be a prime with fixed primitive root $g$. Let $H$ denote the set of (nonzero) $e$ th power residues $(\bmod p)$. For integers $i, j(\bmod p)$, define the cyclotomic number $(i, j)$ of order $e$ to be the number of integers $n$ $(\bmod p)$ for which $n / g^{i}$ and $(1+n) / g^{j}$ are both in $H$. If there exists $\alpha \geqslant 1$ such that every nonzero integer $(\bmod p)$ can be expressed as a difference $(\bmod p)$ of elements of $H$ (resp., $H \cup\{0\}$ ) in exactly $\alpha$ ways, one calls $H$ a difference set (resp. modified difference set ).

E. Lehmer [7] has shown that

$H$ is a difference set if and only if $2 \mid e, 2 \nmid f$, and

$$
(i, 0)=(f-1) / e \text { for all } i=0,1,2, \ldots,(e-2) / 2 \text {, }
$$

and

$$
\begin{aligned}
& H \text { is a modified difference set if and only if } 2 \mid e, 2 \nmid f \text {, and } \\
& 1+(0,0)=(i, 0)=(f+1) / e \text { for all } i=1,2, \ldots,(e-2) / 2 \text {. }
\end{aligned}
$$

In Section 5 of this paper, we use Lehmer's result, a table of cyclotomic numbers of order twenty-four [6], and a formula for Gauss sums of order twenty-four [3, Theorem 3.32] to prove the following theorem.

THEOREM. Suppose that $p=24 f+1$ is a prime such that either 2 is a cubic residue or 3 is a quartic residue $(\bmod p)$. Then the twenty-fourth powers $(\bmod p)$ do not form $a$ difference set or a modified difference set.

2. History. Chowla [4] and Lehmer [7] have constructed $e$ th power residue difference sets and modified difference sets in the cases $e=2,4,8$. The $e$ th power residue difference sets and modified difference sets have been proved nonexistent for all other values of $e \leqslant 24$, except in the following unsolved cases:

(A) $\quad e=20, \quad p \equiv 21(\bmod 40), \quad 5$ nonquartic $(\bmod p)$,

(B) $e=22, \quad p \equiv 23(\bmod 88), 2$ not an eleventh power $(\bmod p)$,

Received March 10, 1982.

1980 Mathematics Subject Classification. Primary 05B10, 12C20; Secondary 10G05.

Key words and phrases. Power residue difference sets, cyclotomic numbers, Gauss and Jacobi sums.

*Author has NSF grant MCS8101860. 
and

(C) $e=24, \quad p \equiv 25(\bmod 48), 2$ noncubic and 3 nonquartic $(\bmod p)$.

See [7] for $e=6$; [13], [14] for $e=10,12$; [9, Theorems 4 and 5] for $e=14,22$; [12], [5] for $e=16$; [2] for $e=18$; and [10] for $e=20$. See also the paper of Berndt and Evans [3, §5] and the books of Baumert [1], Mann [8], and Storer [11].

3. The Tables of Cyclotomic Numbers of Order Twenty-Four. In the sequel we use the notation of Section 1 with $e=24$. Let $\zeta=\exp (2 \pi i / 24)$ and fix a character $\chi$ $(\bmod p)$ of order twenty-four such that $\chi(g)=\zeta$. For characters $\lambda, \Psi(\bmod p)$, define the Jacobi sums

$$
J(\lambda, \Psi)=\sum_{n(\bmod p)} \lambda(n) \Psi(1-n), \quad K(\lambda)=\lambda(4) J(\lambda, \lambda) .
$$

It is known [3, §3] that there exist integers $X, Y, A, B, C, D, U, V$ such that

$$
\begin{array}{ll}
K\left(\chi^{6}\right)=-X+2 Y i & \left(p=X^{2}+4 Y^{2}, X \equiv 1(\bmod 4)\right), \\
K\left(\chi^{4}\right)=-A+B i \sqrt{3} & \left(p=A^{2}+3 B^{2}, A \equiv 1(\bmod 6)\right), \\
K\left(\chi^{3}\right)=-C+D i \sqrt{2} & \left(p=C^{2}+2 D^{2}, C \equiv 1(\bmod 4)\right),
\end{array}
$$

and

$$
K(\chi)=U+2 V i \sqrt{6} \quad\left(p=U^{2}+24 V^{2}, U \equiv-C(\bmod 3)\right) .
$$

Since $J\left(\chi, \chi^{2}\right) \in \mathbf{Z}[\zeta]$, there exist integers $D_{0}, D_{1}, \ldots, D_{7}$ such that

$$
J\left(\chi, \chi^{2}\right)=\sum_{i=0}^{7} D_{i} \zeta^{i} .
$$

In the 48 tables [6], each number $576(i, j)$ has been expressed as a linear combination of $p, 1, X, Y, A, B, C, D, U, V, D_{0}, \ldots, D_{7}$ over $\mathbf{Z}$.

4. Gauss Sums of Order Twenty-Four. Consider the Gauss sum

$$
G_{e}=\sum_{n=0}^{p-1} \exp \left(2 \pi i n^{e} / p\right) \text {. }
$$

Define, for real $\gamma$,

$$
F_{e}(\gamma)=\left|G_{e}+\gamma\right|^{2}-\left(p(e-1)+\gamma^{2}\right) .
$$

It is known [3, p. 391] that, for $e=24$,

(3) $\quad H$ is a difference set (resp., modified difference set) if and only if

$$
F_{24}(-1)=0\left(\text { resp., } F_{24}(23)=0\right) \text {. }
$$

5. Proof of Theorem. By (1) and $\left(1^{\prime}\right)$, we may assume that $f$ is odd. Define $V^{\prime} \in\{0,1\}$ by $V^{\prime} \equiv V(\bmod 2)$. Let $Z=\operatorname{ind} 2(\bmod 12)$ and $T=\operatorname{ind} 3(\bmod 8)$, where the indices are taken with respect to the primitive $\operatorname{root} g(\bmod p)$. We may assume without loss of generality that $Z \in\{0,2,4,6\}$ and $T \in\{0,2,4\}$ (otherwise replace $g$ by an appropriate power of $g$ such as $g^{-1}, g^{5}$, or $g^{7}$ ).

Assume that $H$ is a difference set or a modified difference set. In particular, then, by (1) and $\left(1^{\prime}\right)$, the numbers

$$
\alpha(i)=576(i, 0)
$$


are equal for $1 \leqslant i \leqslant 11$. We will produce a contradiction in each of the nine cases below. The last case is considerably more complicated than the others since it incorporates the results on Gauss sums from Section 4 and [3, Theorem 3.32].

Case 1. $V^{\prime}=Z=0$.

From Tables 25-27 in [6],

$$
\begin{array}{ll}
0=\alpha(1)+\alpha(5)-\alpha(7)-\alpha(11)=192 Y, & \text { if } T=0, \\
0=\alpha(1)+\alpha(7)-\alpha(5)-\alpha(11)=48 B, & \text { if } T=2,
\end{array}
$$

and

$$
0=\alpha(10)-\alpha(2)=48 B, \text { if } T=4 .
$$

Since clearly $Y$ and $B$ are nonzero, this is a contradiction.

Case 2. $V^{\prime}=0, Z=2$.

From Tables 28 and 30,

$$
0=\alpha(11)-\alpha(5)=96 Y, \text { if } T=0,
$$

and

$$
0=\alpha(5)+\alpha(9)+\alpha(1)-\alpha(3)-\alpha(7)-\alpha(11)=288 Y, \text { if } T=4 .
$$

(Note that $T \neq 2$ in this case, since 3 is quartic by hypothesis.)

Case 3. $V^{\prime}=0, Z=4$.

From Tables 31 and 33,

$$
0=\alpha(1)-\alpha(7)=96 Y, \text { if } T=0,
$$

and

$$
0=\alpha(3)+\alpha(7)+\alpha(11)-\alpha(1)-\alpha(5)-\alpha(9)=192 Y, \quad \text { if } T=4 .
$$

Case 4. $V^{\prime}=0, Z=6$.

From Tables 34-36,

$$
\begin{aligned}
& 0=\alpha(3)-\alpha(9)=96 Y, \quad \text { if } T=0, \\
& 0=\alpha(1)+\alpha(8)-\alpha(4)-\alpha(5)=48 B, \quad \text { if } T=2,
\end{aligned}
$$

and

$$
0=\alpha(2)+\alpha(8)-\alpha(4)-\alpha(10)=96 B, \quad \text { if } T=4 .
$$

Case 5. $V^{\prime}=1, Z=2$.

From Tables 40 and 42 ,

$$
0=\alpha(1)-\alpha(7)=96 Y, \quad \text { if } T=0,
$$

and

$$
0=\alpha(1)+\alpha(5)+\alpha(9)-\alpha(3)-\alpha(7)-\alpha(11)=96 Y, \quad \text { if } T=4 .
$$

Case 6. $V^{\prime}=1, Z=6$.

From Tables 46-48,

$$
\begin{aligned}
& 0=\alpha(1)-\alpha(5)=48 B, \quad \text { if } T=0, \\
& 0=\alpha(1)+\alpha(7)-\alpha(5)-\alpha(11)=48 B, \quad \text { if } T=2,
\end{aligned}
$$

and

$$
0=\alpha(4)-\alpha(8)=48 B, \quad \text { if } T=4
$$


Case 7. $V^{\prime}=1, Z=4$.

First suppose that $T=4$. Then from Table $45,14(\alpha(4)+\alpha(8))+5 \alpha(0)=33 p-$ $879-306 A$. By $(1)$ or $\left(1^{\prime}\right)$, the left side above equals $33 p-825$ or $33 p-2121$, respectively. This yields a contradiction in either case.

Finally, suppose that $T=0$. Then from Table $43,0=2 \alpha(7)+2 \alpha(5)+\alpha(2)+$ $5 \alpha(8)-5 \alpha(4)-\alpha(10)-4 \alpha(3)=288 A+72 B$, so $B=-4 A$ and $p=A^{2}+3 B^{2}=$ $49 A^{2}$, which is absurd.

Case 8. $V^{\prime}=1, Z=0, T \neq 0$.

From Tables 38 and 39,

$$
0=\alpha(2)+\alpha(7)-\alpha(10)-\alpha(11)=144 B, \quad \text { if } T=2,
$$

and

$$
0=\alpha(2)+\alpha(8)-\alpha(4)-\alpha(10)=96 B, \quad \text { if } T=4 .
$$

Case 9. $V^{\prime}=1, Z=0, T=0$.

Assume for the moment that $H$ is a difference set rather than a modified difference set. Then by (1),

$$
p-25=\alpha(0)=\alpha(1)=\alpha(3)
$$

From Table 37,

$$
\begin{aligned}
& 0=\alpha(1)-\alpha(5)=48 B+48 D_{4}, \\
& 0=\alpha(0)-\alpha(6)=16 A+8 C-24,
\end{aligned}
$$

and

$$
0=\alpha(2)-\alpha(4)=-16 A-8 C-24 U
$$

By (12)-(14), we have

$$
B=-D_{4}
$$

and

$$
U=-1 \text {. }
$$

From (11), (13), (15), (16), and the formula for $\alpha(1)$ (in Table 37), we obtain

$$
A=13
$$

and

$$
C=-23 \text {. }
$$

From (11), (18), and the formula for $\alpha(3)$,

$$
X=5 \text {. }
$$

From (11), (16), (17), (19), and the formula for $\alpha(0)$,

$$
2 D_{0}+D_{4}=16 \text {. }
$$

Conversely, if equalities (8)-(13) hold, then $H$ is a difference set; this follows easily from (1) and Table 37 . We will see shortly that (8)-(13) cannot all hold. It is interesting to note, however, that (9)-(13) all hold for $p=601$.

By arguing as above, we can show that $H$ is a modified difference set if and only if the following equalities $\left(8^{\prime}\right)-\left(13^{\prime}\right)$ all hold:

$$
\begin{aligned}
& B=-D_{4}, \\
& U=23,
\end{aligned}
$$




$$
\begin{aligned}
A & =-299, \\
C & =529, \\
X & =-115, \\
2 D_{0}+D_{4} & =-368 .
\end{aligned}
$$

Unfortunately we do not see how to obtain contradictions from (8)-(13) or $\left(8^{\prime}\right)-\left(13^{\prime}\right)$ directly from the properties of the Jacobi sums in Section 3. Instead, we obtain contradictions using the results of Section 4 and [3, Theorem 3.32], via the following technical lemma.

Lemma. Suppose that $F_{24}(\gamma)=0$. Then for some $\tau= \pm 1$ and $\nu= \pm 1$,

$$
16(U+\sigma)(\sigma-C)(p+X \sigma)=s^{2}-4 A p q r,
$$

where

$$
\begin{gathered}
\sigma=\sqrt{p}, \quad R=\nu(2 p-2 X \sigma)^{1 / 2}, \quad q=2+(\gamma-X) / \sigma+R(1+\tau) / \sigma, \\
r=2 U-A+\gamma-2 \tau X+R(1+\tau)+2 \sigma(2+\tau)+(\gamma-U) R \tau / \sigma,
\end{gathered}
$$

and

$$
s=-4 p+R(\gamma-2 \tau A+C+2 U)-\sigma(\gamma+2 A+X+2 C-4 U) .
$$

Proof. For brevity, write $G=G_{3}$. Define $T$ as in [3, (3.37)]. By [3, Theorems 3.8 and 3.20], there exists a value of $\nu= \pm 1$ (specifying $R$ ) such that

$$
G_{12}=G+G^{2} / \sigma-\sigma+R+T .
$$

In view of [3, Theorem 3.19], there exists a value of $\tau= \pm 1$ such that

$$
T=\tau G R / \sigma,
$$

since $3 \nmid X$ by $(12),\left(12^{\prime}\right)$. Since $f$ is odd by hypothesis, the expression $W=$ $\pm\left(R_{1}+R_{5}+R_{7}+R_{11}\right)$ given in [3, p. 379] is purely imaginary. Thus, by (15), (16), and [3, Theorem 3.32], we have

$$
\begin{aligned}
G_{24}= & G+G^{2} / \sigma-\sigma+R+\tau G R / \sigma \pm i((2 \sigma-2 C)(2 \sigma-R))^{1 / 2} \\
& \pm i((2 U+2 \sigma)(4 \sigma+2 G+2 R-\tau G R / \sigma))^{1 / 2},
\end{aligned}
$$

where the first five terms on the right of (17) are real and the last two terms are purely imaginary. By (2) and (17), we have, for real $\gamma$,

$$
F_{24}(\gamma)=\left|G_{24}+\gamma\right|^{2}-\gamma^{2}-23 p
$$

so

$$
\begin{aligned}
F_{24}(\gamma)= & -23 p-\gamma^{2}+\left(G+G^{2} / \sigma-\sigma+R+\tau G R / \sigma+\gamma\right)^{2} \\
& +(2 \sigma-2 C)(2 \sigma-R)+(2 U+2 \sigma)(4 \sigma+2 G+2 R-\tau R G / \sigma) \\
& \pm 4 L
\end{aligned}
$$

where

$$
L^{2}=(U+\sigma)(\sigma-C)(2 \sigma-R)(4 \sigma+2 G+2 R-\tau R G / \sigma) .
$$

From [3, Theorem 3.6], since 2 is cubic $(\bmod p)$,

$$
G^{3}=3 p G-2 A p \text {. }
$$


Expanding the right side of (18) and then using (20) to express $G^{3}$ and $G^{4}$ in terms of smaller powers of $G$, we see that

$$
\begin{aligned}
F_{24}(\gamma) \mp 4 L= & -23 p-\gamma^{2}+G^{2}+\left(3 G^{2}-2 A G\right)+p+(2 p-2 X \sigma) \\
& +\left(2 G^{2}-2 G^{2} X / \sigma\right)+\gamma^{2}+(6 \sigma G-4 A \sigma)-2 \sigma G \\
& +2 G R+2 \tau G^{2} R / \sigma+2 G \gamma-2 G^{2}+2 R G^{2} / \sigma \\
& +(6 \tau R G-4 \tau A R)+2 \gamma G^{2} / \sigma-2 \sigma R-2 \tau R G \\
& -2 \sigma \gamma+(4 \tau \sigma G-4 \tau X G)+2 \gamma R+2 \gamma \tau R G / \sigma \\
& +4 p-4 C \sigma-2 \sigma R+2 C R+8 U \sigma+4 U G+4 U R \\
& -2 \tau U R G / \sigma+8 p+4 \sigma G+4 \sigma R-2 \tau R G .
\end{aligned}
$$

Since $F_{24}(\gamma)=0$ by the hypothesis of the Lemma, it follows that

$$
\pm 2 L=q G^{2}+r G+s .
$$

Squaring the right side of (21) and then using (20) to simplify as before, we find that

$$
4 L^{2}=G^{2}\left(r^{2}+2 q s+3 p q^{2}\right)+G\left(6 p q r+2 r s-2 A p q^{2}\right)+\left(s^{2}-4 A p q r\right) .
$$

Now, the degrees of $G$ and $R$ over $\mathbf{Q}$ are 3 and 4 , respectively, and it is consequently easy to see that $G$ has degree 3 over $Q(R)$. From (19), we can express the left side of (22) as a linear polynomial in $G$ over $\mathbf{Q}(R)$ with constant term

$$
16(U+\sigma)(\sigma-C)(p+X \sigma) \text {. }
$$

Since the constant term on the right side of (22) is $s^{2}-4 A p q r$, the Lemma is proved.

Assume that $H$ is a difference set, so that (8)-(13) hold. Then by $(3), F_{24}(-1)=0$, so by the Lemma, (14) holds with $\gamma=-1$. Thus,

$$
16\left(p^{2}+27 p^{3 / 2}+87 p-115 p^{1 / 2}\right)=s^{2}-52 p q r,
$$

where $q, r, s$ are given in the following table:

\begin{tabular}{|c|c|c|c|}
\hline$\tau$ & $q$ & $r$ & $s$ \\
\hline-1 & $2-6 / \sigma$ & $2 \sigma-6$ & $12 \sigma-4 p$ \\
\hline 1 & $2-6 / \sigma+2 R / \sigma$ & $-26+6 \sigma+2 R$ & $12 \sigma-4 p-52 R$ \\
\hline
\end{tabular}

If $\tau=-1$, the right side of (23) equals $16\left(p^{2}-19 p^{3 / 2}+87 p-117 p^{1 / 2}\right)$, which yields a contradiction. If $\tau=1$, we can express the right side of (23) as a linear polynomial in $R$ over $\mathbf{Q}(\sigma)$ and then compare coefficients of $R$ in (23) to obtain the contradiction $0=416\left(5 p^{1 / 2}-p\right)$.

Finally, assume that $H$ is a modified difference set, so that $\left(8^{\prime}\right)-\left(13^{\prime}\right)$ hold. Then by (3), $F_{24}(23)=0$, so by the Lemma, (14) holds with $\gamma=23$. Thus

$$
16\left(p^{2}-621 p^{3 / 2}+46023 p+1399205 p^{1 / 2}\right)=s^{2}+1196 p q r
$$

where $q, r, s$ are given in the following table:

\begin{tabular}{|c|c|c|c|}
\hline$\tau$ & $q$ & $r$ & $s$ \\
\hline-1 & $2+138 / \sigma$ & $138+\sigma$ & $-4 p-276 \sigma$ \\
\hline 1 & $2+138 / \sigma+2 R / \sigma$ & $598+6 \sigma+2 R$ & $-4 p-276 \sigma+1196 R$ \\
\hline
\end{tabular}


If $\tau=-1$, the right side of $\left(23^{\prime}\right)$ equals $16\left(p^{2}+437 p^{3 / 2}+46023 p+1423539 p^{1 / 2}\right)$, which yields a contradiction. If $\tau=1$, comparison of coefficients of $R$ in (23') yields the contradiction $0=9568\left(p+115 p^{1 / 2}\right)$.

Department of Mathematics University of California at San Diego

La Jolla, California 92093

1. L. D. Baumert, Cyclic Difference Sets, Lecture Notes in Math., vol. 182, Springer-Verlag, Berlin, 1971.

2. L. D. Baumert \& H. Fredericksen, "The cyclotomic numbers of order eighteen with applications to difference sets," Math. Comp., v. 21, 1967, pp. 204-219.

3. B. C. BeRnDt \& R. J. Evans, "Sums of Gauss, Jacobi, and Jacobsthal," J. Number Theory, v. 11, 1979, p̀p. 349-398.

4. S. Chowla, “A property of biquadratic residues," Proc. Nat. Acad. Sci. India Sect. A, v. 14, 1944, pp. $45-46$.

5. R. J. Evans, "Bioctic Gauss sums and sixteenth power residue difference sets," Acta Arith., v. 38, 1980, pp. 37-46.

6. R. J. Evans, "Table of cyclotomic numbers of order twenty-four," Math. Comp., v. 35, 1980, pp. 1036-1038; UMT file 12[9.10], $98 \mathrm{pp}$.

7. E. LeHMER, "On residue difference sets," Canad. J. Math., v. 5, 1953, pp. 425-432.

8. H. B. ManN, Addition Theorems, Wiley, New York, 1965.

9. J. B. MUSKat, "The cyclotomic numbers of order fourteen," Acta Arith., v. 11, 1966, pp. 263-279.

10. J. B. Muskat \& A. L. Whiteman, "The cyclotomic numbers of order twenty," Acta Arith., v. 17, 1970 , pp. $185-216$.

11. T. StORER, Cyclotomy and Difference Sets, Markham, Chicago, Ill., 1967.

12. A. L. Whiteman, "The cyclotomic numbers of order sixteen," Trans. Amer. Math. Soc., v. 86, 1957 , pp. 401-413.

13. A. L. Whiteman, The Cyclotomic Numbers of Order Ten, Proc. Sympos. Appl. Math., vol. 10, Amer. Math. Soc., Providence, R.I., 1960, pp. 95-111.

14. A. L. Whiteman, "The cyclotomic numbers of order twelve," Acta Arith., v. 6, 1960, pp. 53-76. 